Pacific

Journal of

Mathematics

COTILTING MODULES OVER TAME HEREDITARY ALGEBRAS

Aslak Bakke Buan and Henning Krause 


\title{
COTILTING MODULES OVER TAME HEREDITARY ALGEBRAS
}

\author{
Aslak Bakke Buan and Henning Krause \\ Dedicated to Raymundo Bautista and Roberto Martinez \\ on the occasion of their sixtieth birthday.
}

\begin{abstract}
For a left noetherian ring $\Lambda$, we establish a bijective correspondence between equivalence classes of cotilting $\Lambda$-modules which are not necessarily finitely generated, and torsion pairs $(\mathcal{T}, \mathcal{F})$ for the category of finitely generated $\Lambda$-modules with $\Lambda \in \mathcal{F}$. In the second part of this paper, we give a complete classification of all cotilting modules over a tame hereditary artin algebra.
\end{abstract}

\section{Introduction.}

The concept of a tilting module was introduced by Brenner and Butler for the category of finitely generated modules over a finite dimensional algebra [5]. More recently, various authors studied tilting and cotilting modules in the category of all modules over arbitrary associative rings. For example, Göbel and Trlifaj classified the cotilting modules over the ring $\mathbb{Z}$ of integers [14].

In this paper we concentrate on the representation theory of a finite dimensional algebra $\Lambda$. Our aim is to show that all cotilting modules are relevant when one studies the category $\bmod \Lambda$ of finitely generated modules. More specifically, we establish a correspondence between cotilting $\Lambda$-modules which are not necessarily finitely generated, and torsion pairs for $\bmod \Lambda$.

In the second part of this paper we give a complete classification of all cotilting modules over a finite dimensional tame hereditary algebra. Note that new cotilting modules which are not equivalent to finitely generated ones arise only if $\Lambda$ is of infinite representation type. The tame hereditary algebras are of infinite type and their modules are fairly well understood. In fact, the category of modules over a tame hereditary algebra shares a lot of properties with the category of modules over a Dedekind domain. It is therefore tempting to choose the tame hereditary algebras to give for the first time a complete classification of all cotilting modules for a finite dimensional algebra of infinite representation type. 
Let us recall from [7] the definition of a cotilting module of injective dimension at most 1 for an associative ring $\Lambda$. We denote by $\operatorname{Mod} \Lambda$ the category of all left $\Lambda$-modules. For a module $T$, let $\operatorname{Prod} T$ be the category of all direct summands in any product of copies of $T$. A $\Lambda$-module $T$ is called cotilting module if the following ${ }^{1}$ hold:

(C1) The injective dimension of $T$ is at most 1.

(C2) $\operatorname{Ext}\left(T^{\alpha}, T\right)=0$ for every cardinal $\alpha$.

(C3) There exists an injective cogenerator $Q$ and an exact sequence $0 \rightarrow$ $T^{\prime \prime} \rightarrow T^{\prime} \rightarrow Q \rightarrow 0$ with $T^{\prime}, T^{\prime \prime}$ in $\operatorname{Prod} T$.

(C4) $\mathrm{T}$ is pure-injective.

By definition, two cotilting modules $T$ and $T^{\prime}$ are equivalent if $\operatorname{Prod} T=$ $\operatorname{Prod} T^{\prime}$.

We have the following general result about cotilting modules:

Theorem A. Let $\Lambda$ be a left noetherian ring. There exists a bijection between:

- Torsion pairs $(\mathcal{T}, \mathcal{F})$ for $\bmod \Lambda$ such that $\Lambda \in \mathcal{F}$, and

- equivalence classes of cotilting modules.

A torsion pair $(\mathcal{T}, \mathcal{F})$ corresponding to a cotilting module $T$ satisfies $\mathcal{F}=$ $\{X \in \bmod \Lambda \mid \operatorname{Ext}(X, T)=0\}$.

From now on assume that $\Lambda$ is a tame hereditary finite dimensional algebra [18]. In order to formulate the classification of all cotilting modules we need to recall a few well-known facts. We denote by $\mathcal{R}$ the category of finitely generated regular modules. This is an abelian category and we write $\mathbb{P}$ for the set of isoclasses of simple objects in $\mathcal{R}$. For each $S \in \mathbb{P}$, let $[S]$ denote the equivalence class of $S$ with respect to the smallest equivalence relation on $\mathbb{P}$ satisfying $S \sim S^{\prime}$ if $\operatorname{Ext}\left(S, S^{\prime}\right) \neq 0$. For each $S \in \mathbb{P}$ and $n \in \mathbb{N}$, let $S_{n}$ be the unique indecomposable object in $\mathcal{R}$ of length $n$ satisfying $\operatorname{Hom}\left(S, S_{n}\right) \neq 0$, and $S_{-n}$ denotes the unique indecomposable object of length $n$ satisfying $\operatorname{Hom}\left(S_{-n}, S\right) \neq 0$. There are chains of monomorphisms $S=S_{1} \rightarrow S_{2} \rightarrow \ldots$ and chains of epimorphisms $\cdots \rightarrow S_{-2} \rightarrow S_{-1}=S$ for each $S \in \mathbb{P}$. The corresponding Prüfer module is the colimit $S_{\infty}=\lim _{\longrightarrow} S_{n}$ whereas the adic module is $S_{-\infty}=\lim _{-n}$ (which is often denoted by $\widehat{S}$ ). Moreover, there is a unique generic module $G$, that is, $G$ is indecomposable of infinite length and has finite length over $\operatorname{End}(G)$.

For a module $M$, we denote by indec $M$ the set of isoclasses of indecomposable direct summands of $M$. If $M$ is pure-injective, then there is a unique family $\left(M_{i}\right)_{i \in I}$ of modules $M_{i} \in \operatorname{indec} M$ such that $M$ is the pure-injective envelope of $\coprod_{i} M_{i}$. The well-known classification of the indecomposable pure-injectives over a tame hereditary algebra allows us to classify all cotilting modules.

\footnotetext{
${ }^{1}$ Recently, Silvana Bazzoni has shown that (C1)-(C3) implies (C4).
} 
Theorem B. Let $\Lambda$ be a tame hereditary algebra and let $T$ be a pureinjective $\Lambda$-module.

(1) Suppose all indecomposable direct summands are finitely generated. Then $T$ is a cotilting module if and only if the number of non-isomorphic indecomposable direct summands of $T$ equals the number of simple $\Lambda$-modules and $\operatorname{Ext}\left(T^{\prime}, T^{\prime \prime}\right)=0$ for all $T^{\prime}, T^{\prime \prime} \in \operatorname{indec} T$.

(2) Suppose there is an indecomposable direct summand which is not finitely generated. Then $T$ is a cotilting module if and only if the following hold:

- Each indecomposable direct summand of $T$ is either generic or of the form $S_{n}$ for some $S \in \mathbb{P}$ and some $n \in \mathbb{N} \cup\{-\infty, \infty\}$.

- For each $S \in \mathbb{P}$, let $I_{S}$ be the set of non-isomorphic indecomposable direct summands of $T$ which are of the form $S_{n}^{\prime}$ for some $n \in \mathbb{N} \cup\{-\infty, \infty\}$ and some $S^{\prime} \in[S]$. Then $\operatorname{card} I_{S}=\operatorname{card}[S]$ and $\operatorname{Ext}\left(T^{\prime}, T^{\prime \prime}\right)=0$ for all $T^{\prime}, T^{\prime \prime} \in I_{S}$.

(3) Two cotilting modules $T_{1}$ and $T_{2}$ are equivalent if and only if indec $\left(T_{1} \amalg\right.$ $G)=\operatorname{indec}\left(T_{2} \amalg G\right)$.

The paper is organized as follows: In Section 1 we establish the bijective correspondence between cotilting modules and torsion pairs. In addition, we include a generalization to locally noetherian Grothendieck categories. From Section 2 onwards, we restrict ourselves to tame hereditary algebras. First, we recall briefly the classification of the pure-injective modules. Then we describe the selforthogonal pure-injective modules. A cotilting module is a selforthogonal module which is maximal with respect to this property. This observation is the basis of our classification of all cotilting modules which is completed in Section 3. Each cotilting module which is not equivalent to a finitely generated one is the pure-injective envelope of a direct sum $\coprod_{\mathcal{T}} T_{\mathcal{T}}$ where $\mathcal{T}$ runs through the set of tubes in the category of regular modules. Each summand $T_{\mathcal{T}}$ can be viewed as a cotilting object in some appropriate Grothendieck category, and a detailed description is given in the final Section 4.

\section{A correspondence for cotilting modules.}

Let $\Lambda$ be an associative $k$-algebra over some commutative ring $k$. Throughout we assume that $\Lambda$ is left noetherian. Let $\operatorname{Mod} \Lambda$ be the category of (left) $\Lambda$-modules, and let mod $\Lambda$ denote the full subcategory which is formed by all finitely generated modules. To simplify our notation, we write $\operatorname{Hom}(-,-)$ and $\operatorname{Ext}(-,-)$ for the functors $\operatorname{Hom}_{\Lambda}(-,-)$ and $\operatorname{Ext}_{\Lambda}^{1}(-,-)$, respectively. We fix a minimal injective cogenerator $I$ for $\operatorname{Mod} k$ and write $D=\operatorname{Hom}_{k}(-$, $I)$ for the usual duality between left and right $\Lambda$-modules. 
Now fix a $\Lambda$-module $M$. We define $\operatorname{Prod} M$ to be the full subcategory of all direct summands of products of copies of $M$. The full subcategory formed by all submodules of modules in $\operatorname{Prod} M$ is denoted by Cogen $M$. The perpendicular category of $M$ is by definition the full subcategory ${ }^{\perp} M=\{X \in \operatorname{Mod} \Lambda \mid \operatorname{Ext}(X, M)=0\}$. We call $M$ selforthogonal (more precisely, product-selforthogonal) if $\operatorname{Ext}\left(M^{\alpha}, M\right)=0$ for every cardinal $\alpha$. A selforthogonal module $M$ is maximal selforthogonal if $\operatorname{Prod} M \subseteq \operatorname{Prod} N$ implies $\operatorname{Prod} M=\operatorname{Prod} N$ for every selforthogonal module $N$.

Let $\mathcal{C} \subseteq \bmod \Lambda$ be a subcategory. Then we denote by $\lim _{\longrightarrow} \mathcal{C}$ the full subcategory formed by the filtered colimits of objects in $\mathcal{C}$.

1.1. A correspondence. In this section we establish a correspondence between cotilting modules and certain torsion pairs for $\bmod \Lambda$. We start with some preparations.

Lemma 1.1. Let $\mathcal{X} \subseteq \operatorname{Mod} \Lambda$ be a subcategory which is closed under taking subobjects and filtered colimits. Let $\mathcal{C}=\mathcal{X} \cap \bmod \Lambda$. Then $\mathcal{X}=\lim _{\longrightarrow} \mathcal{C}$.

Proof. Clear, since every module is a filtered colimit of its finitely generated submodules.

Recall that a torsion pair $(\mathcal{T}, \mathcal{F})$ for an abelian category $\mathcal{A}$ is a pair of full subcategories such that $\operatorname{Hom}(T, F)=0$ for all $T \in \mathcal{T}, F \in \mathcal{F}$, and every object $X \in \mathcal{A}$ has a subobject $t X \in \mathcal{T}$ with $X / t X \in \mathcal{F}$.

Lemma 1.2. Let $(\mathcal{T}, \mathcal{F})$ be a torsion pair for $\bmod \Lambda$. Then $\left(\underline{\lim } \mathcal{T}, \lim _{\longrightarrow} \mathcal{F}\right)$ is a torsion pair for $\operatorname{Mod} \Lambda$.

Proof. See $[\mathbf{9}]$.

We need to recall some more terminology. To this end fix a category $\mathcal{A}$ and some subcategory $\mathcal{X}$. Given an object $M$ in $\mathcal{A}$, a map $X \rightarrow M$ is called right $\mathcal{X}$-approximation of $M$ provided that $X$ belongs to $\mathcal{X}$ and the induced map $\operatorname{Hom}\left(X^{\prime}, X\right) \rightarrow \operatorname{Hom}\left(X^{\prime}, M\right)$ is surjective for all $X^{\prime}$ in $\mathcal{X}$. A right approximation $f: X \rightarrow M$ is minimal if every endomorphism $g: X \rightarrow X$ satisfying $f \circ g=f$ is an isomorphism. The subcategory $\mathcal{X}$ is contravariantly finite if every object in $\mathcal{A}$ admits a right $\mathcal{X}$-approximation.

Lemma 1.3. Let $\mathcal{X} \subseteq \operatorname{Mod} \Lambda$ be a subcategory which is closed under taking subobjects, extensions, products, and filtered colimits. Then every $\Lambda$-module $M$ has a minimal right $\mathcal{X}$-approximation $M_{\mathcal{X}} \rightarrow M$. The kernel of this approximation is pure-injective. Moreover, $M_{\mathcal{X}}$ is pure-injective provided that $M$ is pure-injective.

Proof. We use an idea from $[\mathbf{1 6}$, Section 2]. Choose a left $D \mathcal{X}$-approximation $D M \rightarrow D X$ which exists by [16, Lemma 2.1], and denote by $C$ its cokernel. 
Apply the duality and take the pullback along the natural map $M \rightarrow D^{2} M$ :

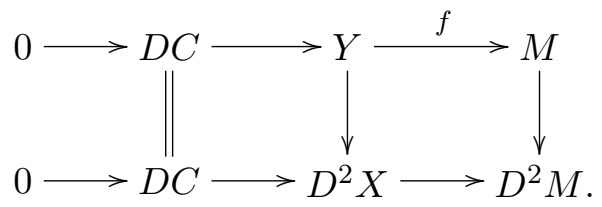

Note that $Y$ belongs to $\mathcal{X}$ since $D^{2} \mathcal{X} \subseteq \mathcal{X}$ by [16, Lemma 4.4]. The construction implies that $f$ is a right $\mathcal{X}$-approximation of $M$. A minimal right $\mathcal{X}$-approximation $M_{\mathcal{X}} \rightarrow M$ exists since $\mathcal{X}$ is closed under filtered colimits; see $[\mathbf{1 1}$, p. 207]. It is well-known that any module of the form $D X$ is pureinjective. Therefore the kernel of $M_{\mathcal{X}} \rightarrow M$ is pure-injective since it is a direct summand of $D C$. The natural map $M \rightarrow D^{2} M$ is a pure monomorphism. This implies the map splits if $M$ is pure-injective. In this case $M_{\mathcal{X}}$ is a direct summand of $D^{2} X$ and therefore pure-injective.

The next proposition describes a construction for cotilting modules. In [2], a similar result is proved under the additional hypothesis that $\mathcal{X}$ is contravariantly finite.

Proposition 1.4. Let $\mathcal{X} \subseteq \operatorname{Mod} \Lambda$ be a subcategory which is closed under taking subobjects, extensions, products, and filtered colimits. Suppose in addition $\Lambda \in \mathcal{X}$. Then there exists a $\Lambda$-module $T$ having the following properties:

(1) There exists an exact sequence $0 \rightarrow T^{\prime \prime} \rightarrow T^{\prime} \rightarrow Q \rightarrow 0$ such that $T=T^{\prime} \amalg T^{\prime \prime}$ and $Q$ is an injective cogenerator;

(2) $T$ is pure-injective and $\operatorname{id} T \leq 1$;

(3) $\mathcal{X}=\operatorname{Cogen} T={ }^{\perp} T$;

(4) $\mathcal{X} \cap \mathcal{X}^{\perp}=\operatorname{Prod} T$.

Proof. (1) We apply Lemma 1.3 to construct $T$. Choose an injective cogenerator $Q$ and let $0 \rightarrow T^{\prime \prime} \rightarrow T^{\prime} \rightarrow Q \rightarrow 0$ be an exact sequence such that $T^{\prime} \rightarrow Q$ is a minimal right $\mathcal{X}$-approximation. Define $T=T^{\prime} \amalg T^{\prime \prime}$. Note that $T^{\prime \prime} \in \mathcal{X}^{\perp}$ by Wakamatsu's lemma. Thus $T \in \mathcal{X}^{\perp}$.

(2) $T$ is pure-injective by Lemma 1.3. To show id $T \leq 1$, take an arbitrary $\Lambda$-module $M$ and choose an exact sequence $0 \rightarrow X_{1} \rightarrow X_{0} \rightarrow M \rightarrow 0$ with $X_{i} \in \mathcal{X}$ which exists by Lemma 1.3. Now apply $\operatorname{Hom}(-, T)$ to see that $\operatorname{Ext}^{2}(M, T)=0$ since $\operatorname{Ext}(\mathcal{X}, T)=0$.

(3) Let $M$ be a $\Lambda$-module and $M \rightarrow Q^{\alpha}$ a monomorphism. The map $M \rightarrow Q^{\alpha}$ factors through the map $\left(T^{\prime}\right)^{\alpha} \rightarrow Q^{\alpha}$ if $M \in{ }^{\perp} T$. Therefore ${ }^{\perp} T \subseteq$ Cogen $T$. We have Cogen $T \subseteq \mathcal{X}$ since $T$ belongs to $\mathcal{X}$ and $\mathcal{X}$ is closed under taking subobjects and products. Finally, $\mathcal{X} \subseteq{ }^{\perp} T$ since $T \in \mathcal{X}^{\perp}$.

(4) We have Prod $T \subseteq \mathcal{X} \cap \mathcal{X}^{\perp}$ since $\mathcal{X}$ and $\mathcal{X}^{\perp}$ are closed under products. Let $M \in \mathcal{X} \cap \mathcal{X}^{\perp}$. Choose a left $\operatorname{Prod} T$-approximation $f: M \rightarrow T^{\alpha}$ which 
is a monomorphism since $\mathcal{X}=\operatorname{Cogen} T$. Now apply $\operatorname{Hom}(-, T)$ to see that Coker $f$ belongs to ${ }^{\perp} T=\mathcal{X}$. Thus $M \rightarrow T^{\alpha}$ splits.

Theorem 1.5. Let $\Lambda$ be a left noetherian ring. There exists a bijection between:

- Torsion pairs $(\mathcal{T}, \mathcal{F})$ for $\bmod \Lambda$ such that $\Lambda \in \mathcal{F}$, and

- equivalence classes of cotilting modules.

$A$ cotilting module $T$ corresponding to a torsion pair $(\mathcal{T}, \mathcal{F})$ satisfies

$$
\operatorname{Prod} T=\left(\lim _{\longrightarrow} \mathcal{F}\right) \cap\left(\lim _{\longrightarrow} \mathcal{F}\right)^{\perp} \quad \text { and } \quad{ }^{\perp} T \cap \bmod \Lambda=\mathcal{F} .
$$

Remark 1.6. Suppose $\Lambda$ is an artin algebra. Then a cotilting module $T$ is equivalent to a finitely generated cotilting module if and only if $\mathcal{F}=$ ${ }^{\perp} T \cap \bmod \Lambda$ is contravariantly finite in $\bmod \Lambda$. This follows from the correspondence for cotilting modules in $[\mathbf{3}]$.

Proof of Theorem 1.5. Let $(\mathcal{T}, \mathcal{F})$ be a torsion pair for $\bmod \Lambda$. Then $\mathcal{X}=$ $\lim _{\longrightarrow} \mathcal{F}$ is closed under taking subobjects, extensions, products, and filtered colimits by Lemma 1.2. Thus we get a cotilting module $T$ satisfying $\operatorname{Prod} T$ $=\mathcal{X} \cap \mathcal{X}^{\perp}$ by Proposition 1.4.

Conversely, let $T$ be a cotilting module. Then ${ }^{\perp} T=\underline{\lim }_{\longrightarrow} \mathcal{F}$ for $\mathcal{F}=$ ${ }^{\perp} T \cap \bmod \Lambda$ by Lemma 1.1. Thus we obtain a torsion pair $(\mathcal{T}, \mathcal{F})$ for $\bmod \Lambda$ since $\mathcal{X}$ is a torsion-free class for $\operatorname{Mod} \Lambda$. It is straightforward to check that the maps $(\mathcal{T}, \mathcal{F}) \mapsto T$ and $T \mapsto(\mathcal{T}, \mathcal{F})$ are mutually inverse.

1.2. Perpendicular categories. Our next aim is to show that ${ }^{\perp} M$ is closed under taking products for a pure-injective module $M$ with id $M \leq 1$. For this we need to assume that $\Lambda$ is left artinian. We start with some preparations.

Lemma 1.7. Let $\mathcal{F} \subseteq \bmod \Lambda$ be a subcategory which is closed under taking subobjects and extensions. Let $\mathcal{T}$ be the subcategory formed by all $M \in$ $\bmod \Lambda$ satisfying $\operatorname{Hom}(M, \mathcal{F})=0$. Then $(\mathcal{T}, \mathcal{F})$ is a torsion pair for $\bmod \Lambda$.

Proof. Each finitely generated module $M$ has a minimal submodule $t M$ such that $M / t M$ belongs to $\mathcal{F}$. For this we use that $M$ is artinian and that $\mathcal{F}$ is closed under subobjects. The submodule $t M$ belongs to $\mathcal{T}$ since $\mathcal{F}$ is closed under extensions.

Each torsion-free class in $\operatorname{Mod} \Lambda$ is closed under taking products. We have therefore the following consequence:

Proposition 1.8. Let $\Lambda$ be left artinian. Suppose $\mathcal{X} \subseteq \operatorname{Mod} \Lambda$ is a subcategory which is closed under taking subobjects, extensions, and filtered colimits. Then $\mathcal{X}$ is closed under taking products.

Proof. Let $\mathcal{F}=\mathcal{X} \cap \bmod \Lambda$. Then we have $\mathcal{X}=\lim _{\longrightarrow} \mathcal{F}$ by Lemma 1.1, which is a torsion-free class by Lemma 1.7 and 1.2. Thus $\mathcal{X}$ is closed under products. 
Our application about perpendicular categories is based on the following well-known fact:

Lemma 1.9. Let $\left\{M_{i}\right\}$ be a filtered system of modules and $N$ be a pureinjective module. Then $\operatorname{Ext}\left(\lim _{\longrightarrow} M_{i}, N\right) \cong \lim _{\operatorname{Ext}} \operatorname{E}\left(M_{i}, N\right)$.

Corollary 1.10. Let $\Lambda$ be left artinian. Suppose $M$ is a pure-injective module of injective dimension at most 1 . Denote by $\mathcal{F}$ the class of finitely generated modules in ${ }^{\perp} M$. Then ${ }^{\perp} M=\underline{\lim _{1}} \mathcal{F}$. Moreover, ${ }^{\perp} M$ is closed under taking products.

1.3. Complements. Next we discuss when a selforthogonal module $T$ has a complement $T^{\prime}$ such that $T \amalg T^{\prime}$ is a cotilting module. This problem has been studied before, for instance in [1]. Here we use Corollary 1.10 and get some new result. We need the following well-known lemma which is based on a construction due to Bongartz:

Lemma 1.11. Let $T$ be a selforthogonal module and $Q$ be an injective cogenerator. Suppose ${ }^{\perp} T$ is closed under taking products. Then there exists a module $T^{\prime}$ having the following properties:

(1) There exists an exact sequence $0 \rightarrow T^{\alpha} \rightarrow T^{\prime} \rightarrow Q \rightarrow 0$ for some cardinal $\alpha$.

(2) $T \amalg T^{\prime}$ is selforthogonal and ${ }^{\perp} T={ }^{\perp}\left(T \amalg T^{\prime}\right)$.

(3) If $T$ is pure-injective, then $T^{\prime}$ is pure-injective.

Proof. We sketch the proof for the convenience of the reader. The crucial idea is that of a universal extension $[4,8]$. That is, take the product of all exact sequences in $\operatorname{Ext}(Q, T)$ and let $\alpha=\operatorname{card}(\operatorname{Ext}(Q, T))$. Now take the pullback along the codiagonal map $Q \rightarrow Q^{\alpha}$, to obtain a sequence

$$
0 \longrightarrow T^{\alpha} \longrightarrow T^{\prime} \longrightarrow Q \longrightarrow 0 \text {. }
$$

Clearly, ${ }^{\perp} T={ }^{\perp}\left(T \amalg T^{\prime}\right)$. The construction of the universal extension implies $\operatorname{Ext}\left(T^{\prime}, T\right)=0$. Thus $T \amalg T^{\prime}$ is selforthogonal.

The last assertion follows from the following fact: A module $M$ satisfying (C1)-(C3) in the definition of a cotilting module is pure-injective if and only if ${ }^{\perp} M$ is closed under taking filtered colimits and pure submodules; see Proposition 5.7 in [16].

Corollary 1.12. Let $\Lambda$ be left artinian. Suppose $T$ is a pure-injective module of injective dimension at most 1 satisfying $\operatorname{Ext}(T, T)=0$. Then there exists a module $T^{\prime}$ such that $T \amalg T^{\prime}$ is a cotilting module.

1.4. A generalization. The correspondence in Theorem 1.5 can be generalized as follows: Let $\mathcal{A}$ be a locally noetherian Grothendieck category, that is, $\mathcal{A}$ is a Grothendieck category and has a generating set of noetherian objects. Recall that a set $\mathcal{G}$ of objects generates an additive category $\mathcal{C}$ if for every nonzero map $f$ in $\mathcal{C}$ we have $\operatorname{Hom}(G, f) \neq 0$ for some $G \in \mathcal{G}$. Next we 
recall that an object $X$ in $\mathcal{A}$ is pure-injective if every pure exact sequence $\varepsilon: 0 \rightarrow X \rightarrow Y \rightarrow Z \rightarrow 0$ splits. Here, $\varepsilon$ is pure exact if $\operatorname{Hom}(A, \varepsilon)$ is an exact sequence for every noetherian object $A$ in $\mathcal{A}$. Finally, we say that an object $T$ in $\mathcal{A}$ is a cotilting object if $T$ satisfies the conditions (C1)-(C4).

Theorem 1.13. Let $\mathcal{A}$ be a locally noetherian Grothendieck category and denote by noeth $\mathcal{A}$ the full subcategory formed by all noetherian objects. There exists a bijection between:

- Torsion pairs $(\mathcal{T}, \mathcal{F})$ for noeth $\mathcal{A}$ such that $\mathcal{F}$ generates noeth $\mathcal{A}$, and - equivalence classes of cotilting objects.

A cotilting object $T$ corresponding to a torsion pair $(\mathcal{T}, \mathcal{F})$ satisfies

$$
\operatorname{Prod} T=(\underline{\lim } \mathcal{F}) \cap(\underline{\lim } \mathcal{F})^{\perp} \quad \text { and } \quad{ }^{\perp} T \cap \operatorname{noeth} \mathcal{A}=\mathcal{F} .
$$

Proof. The proof is essentially the same as that of Theorem 1.5. However, it remains to explain the analogue of the duality $D$ between left and right modules which is used in the proof of Lemma 1.3. To this end let $\mathcal{C}=$ noeth $\mathcal{A}$ and denote by $\left(\mathcal{C}^{\mathrm{op}}, \mathrm{Ab}\right)$ the category of additive functors $F: \mathcal{C}^{\mathrm{op}} \rightarrow$ Ab. The functor

$$
\mathcal{A} \longrightarrow\left(\mathcal{C}^{\mathrm{op}}, \mathrm{Ab}\right),\left.\quad X \mapsto \operatorname{Hom}(-, X)\right|_{\mathcal{C}},
$$

identifies $\mathcal{A}$ with the full subcategory of left exact functors $\mathcal{C}^{\text {op }} \rightarrow \mathrm{Ab}$; see $\left[\mathbf{1 2}\right.$. The duality $D=\operatorname{Hom}_{\mathbb{Z}}(-, \mathbb{Q} / \mathbb{Z})$ induces a duality between $\left(\mathcal{C}^{\text {op }}, \mathrm{Ab}\right)$ and $(\mathcal{C}, \mathrm{Ab})$ via the assignment $F \mapsto D \circ F$. It is straightforward to check that the proof of Lemma 1.3 works with this duality.

\section{Selforthogonal modules.}

Throughout the rest of this paper we assume that $\Lambda$ is a tame hereditary algebra. We refer to Ringel's Rome notes [18] for basic facts about the representation theory of tame hereditary algebras. In this section we discuss pure-injective $\Lambda$-modules which are selforthogonal. A basic tool are the following Auslander-Reiten formulas:

$$
D \operatorname{Ext}(X, M) \cong \operatorname{Hom}(M, \tau X) \text { and } \operatorname{Ext}(M, X) \cong D \operatorname{Hom}\left(\tau^{-1} X, M\right)
$$

which are valid for every $\Lambda$-module $M$ and every finitely generated module $X$; see [10]. Here, $\tau X$ denotes the dual of transpose of $X$.

2.1. Pure-injectives. Let Ind $\Lambda$ denote the set of isoclasses of indecomposable pure-injective $\Lambda$-modules and let ind $\Lambda$ be the set of finitely generated objects in Ind $\Lambda$. The classification of indecomposable pure-injectives is wellknown:

$$
\text { Ind } \Lambda=\operatorname{ind} \Lambda \cup\left\{S_{\infty} \mid S \in \mathbb{P}\right\} \cup\left\{S_{-\infty} \mid S \in \mathbb{P}\right\} \cup\{G\} .
$$

The category of finitely generated modules decomposes into three subcategories: The preprojectives $\mathcal{P}$, the preinjectives $\mathcal{I}$, and the regular modules 
$\mathcal{R}$. The category $\mathcal{R}$ is abelian and a $\Lambda$-module is called quasi-simple if it is a simple object in $\mathcal{R}$. The following picture indicates the direction of nonzero maps between indecomposable pure-injectives:

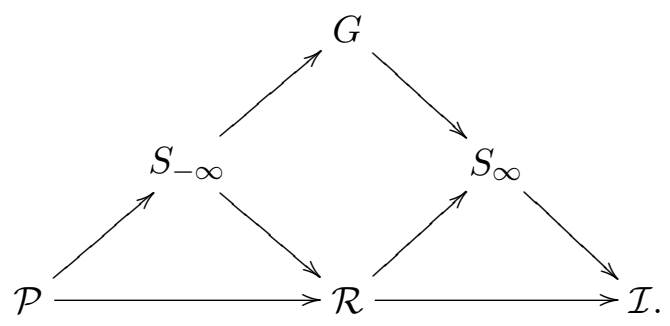

Proposition 2.1. Let $M$ be a pure-injective module. Then there exists a family of indecomposable pure-injective modules $\left(M_{i}\right)_{i \in I}$ such that $M$ is a pure-injective envelope of $\coprod_{i \in I} M_{i}$. The $M_{i}$ are unique up to isomorphism; they are, up to isomorphism, precisely the indecomposable direct summands of $M$.

Proof. See Proposition 8.33 and Theorem 8.53 in [15].

The description of the pure-injectives has some useful consequences.

Lemma 2.2. Let $M$ be a pure-injective module. then ${ }^{\perp} M=\bigcap_{N}{ }^{\perp} N$ where $N$ runs through all indecomposable direct summands of $M$.

Proof. This follows immediately from Proposition 2.1. Write $M=E\left(\coprod_{i} M_{i}\right)$ as the pure-injective envelope of a coproduct of indecomposables. Then we have

$$
\bigcap_{i}^{\perp} M_{i}={ }^{\perp}\left(\prod_{i} M_{i}\right) \subseteq{ }^{\perp} M \subseteq \bigcap_{i}{ }^{\perp} M_{i}
$$

since $M$ is a direct summand of $\prod_{i} M_{i}$.

The following result simplifies the description of the pure-injective $\Lambda$ modules which are selforthogonal: 
Corollary 2.3. For a pure-injective module $M$ the following are equivalent:

(1) $\operatorname{Ext}(M, M)=0$;

(2) $\operatorname{Ext}\left(M^{\prime}, M^{\prime \prime}\right)=0$ for all indecomposable direct summands $M^{\prime}, M^{\prime \prime}$ of $M$;

(3) $M$ is selforthognal, that is, $\operatorname{Ext}\left(M^{\alpha}, M\right)=0$ for every cardinal $\alpha$.

Proof. We know from Corollary 1.10 that ${ }^{\perp} M$ is closed under taking products. Writing $M=E\left(\coprod_{i} M_{i}\right)$ as the pure-injective envelope of a coproduct of indecomposables, we see that ${ }^{\perp} M$ contains $M$ since $M$ is a direct summand of $\prod_{i} M_{i}$.

2.2. Extensions between indecomposables. We construct non-split extensions between some indecomposable pure-injectives.

Lemma 2.4. Let $S$ be a quasi-simple module, then there is a (non-split) exact sequence

$$
0 \longrightarrow(\tau S)_{-\infty} \longrightarrow \coprod G \longrightarrow S_{\infty} \longrightarrow 0
$$

Proof. Let $r \in \mathbb{N}$ be the minimal number such that $\tau^{r} S=S$. There is a system of exact sequences

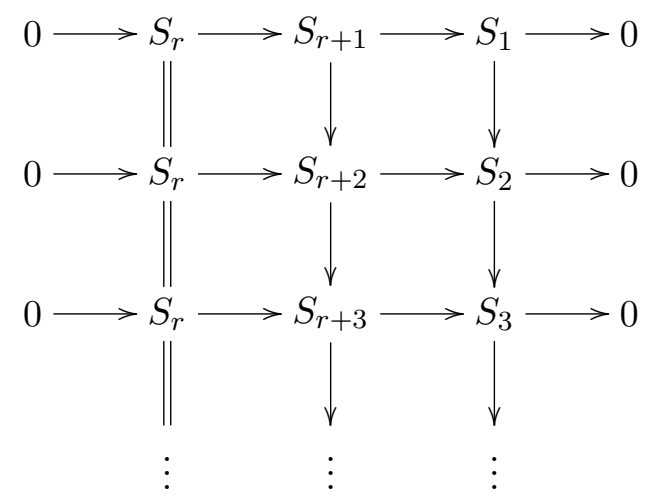

which induces an exact sequence $0 \rightarrow S_{r} \rightarrow S_{\infty} \stackrel{f}{\rightarrow} S_{\infty} \rightarrow 0$ with Ker $f^{n}=$ $S_{n r}$ for all $n \in \mathbb{N}$. It is well-known that the inverse limit of the system

$$
\cdots \stackrel{f}{\longrightarrow} S_{\infty} \stackrel{f}{\longrightarrow} S_{\infty} \stackrel{f}{\longrightarrow} S_{\infty}
$$


is a coproduct of copies of $G$. Using the formula $S_{n r}=(\tau S)_{-n r}$, we obtain the following system of exact sequences:

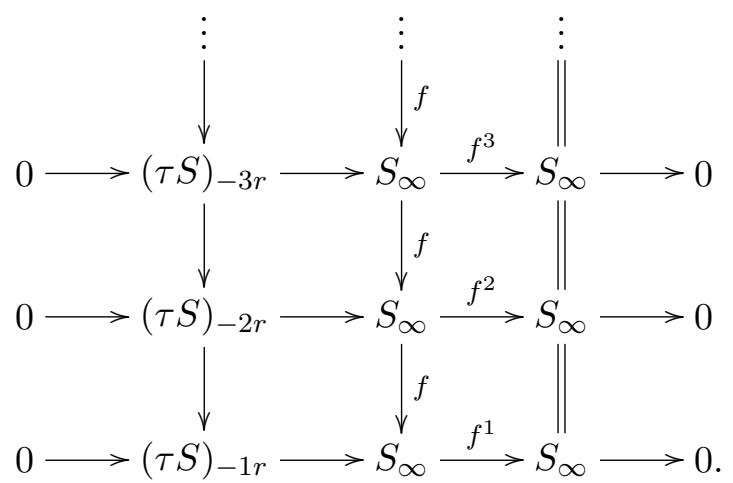

Take inverse limits to obtain the required sequence. It is exact because the system is surjective, and thus the Mittag-Leffler condition (see e.g., [19]) is satisfied.

Lemma 2.5. Let $M$ be an indecomposable pure-injective module which is not finitely generated.

(1) $\operatorname{Ext}(M, Q) \neq 0$ for every nonzero preprojective module $Q$.

(1) $\operatorname{Ext}(J, M) \neq 0$ for every nonzero preinjective module $J$.

Proof. First we assume $M=S_{\infty}$. It is well-known that for any preprojective module $Q$ there is a nonzero map to a module in every tube. Thus there is a nonzero map $\tau^{-1} Q \rightarrow S_{r}^{\prime} \rightarrow S_{\infty}^{\prime}$ for some quasi-simple $S^{\prime} \sim S$. The Auslander-Reiten formula implies $\operatorname{Ext}\left(S_{\infty}^{\prime}, Q\right) \neq 0$. Suppose $S=\tau^{n} S^{\prime}$. There exists an exact sequence $0 \rightarrow S_{n} \rightarrow S_{\infty} \rightarrow S_{\infty}^{\prime} \rightarrow 0$ which induces a monomorphism $\operatorname{Ext}\left(S_{\infty}^{\prime}, Q\right) \rightarrow \operatorname{Ext}\left(S_{\infty}, Q\right)$ since $\operatorname{Hom}\left(S_{n}, Q\right)=0$. Thus $\operatorname{Ext}\left(S_{\infty}, Q\right) \neq 0$.

Now let $J$ be preinjective and suppose there is a nonzero map $S_{r}^{\prime} \rightarrow \tau J$ for some quasi-simple $S^{\prime} \sim S$. We find an epimorphism $S_{n} \rightarrow S_{r}^{\prime}$ for some $n \in \mathbb{N}$. Thus $\operatorname{Ext}\left(J, S_{n}\right) \neq 0$ by the Auslander-Reiten formula. The exact sequence $0 \rightarrow S_{n} \rightarrow S_{\infty} \rightarrow\left(\tau^{-n} S\right)_{\infty} \rightarrow 0$ induces a monomorphism $\operatorname{Ext}\left(J, S_{n}\right) \rightarrow$ $\operatorname{Ext}\left(J, S_{\infty}\right)$ since $\operatorname{Hom}\left(J,\left(\tau^{-n} S\right)_{\infty}\right)=0 . \operatorname{Thus} \operatorname{Ext}\left(J, S_{\infty}\right) \neq 0$.

The statements for $S_{-\infty}$ follow from the statements for $S_{\infty}$ by duality. More precisely, we have $S_{-\infty}=D\left((D S)_{\infty}\right)$, and we combine this fact with the formula $\operatorname{Ext}(M, D N) \cong \operatorname{Ext}(N, D M)$ which is valid for all modules $M$ and $N$.

It remains to consider the generic module $G$. This case reduces to the previous cases since the exact sequence (2.1) induces monomorphisms $\operatorname{Ext}\left(S_{\infty}, Q\right) \rightarrow \operatorname{Ext}(\coprod G, Q)$ and $\operatorname{Ext}\left(J,(\tau S)_{-\infty}\right) \rightarrow \operatorname{Ext}(J, \coprod G)$.

Next we compute the perpendicular categories for Prüfer modules and the generic module. 
Lemma 2.6. Let $M$ be the generic or a Prüfer module. Then ${ }^{\perp} M=$ $\lim _{\longrightarrow}(\mathcal{P} \cup \mathcal{R})$.

Proof. There is a torsion pair $(\mathcal{I}, \mathcal{P} \cup \mathcal{R})$ for the category of finitely generated $\Lambda$-modules. We have that $\mathcal{P}$ and $\mathcal{R}$ belong to ${ }^{\perp} M$, whereas $\mathcal{I} \cap{ }^{\perp} M=0$ by Lemma 2.5. Thus ${ }^{\perp} M=\underset{\lim }{\longrightarrow}(\mathcal{P} \cup \mathcal{R})$ by Corollary 1.10 .

We need to know when $\operatorname{Ext}(M, N)$ vanishes for indecomposable pureinjective modules $M$ and $N$.

Lemma 2.7. Let $M$ and $N$ be indecomposable pure-injective $\Lambda$-modules which are not finitely generated. Then $\operatorname{Ext}(M, N) \neq 0$ if and only if there are quasi-simples $S \sim S^{\prime}$ in $\mathbb{P}$ such that $M \cong S_{\infty}$ and $N \cong S_{-\infty}^{\prime}$.

Proof. Suppose first that $S \sim S^{\prime}$ are quasi-simples in $\mathbb{P}$. The exact sequence (2.1) induces an injective map

$$
\operatorname{Hom}\left(\tau S_{\infty}, S_{\infty}^{\prime}\right) \longrightarrow \operatorname{Ext}\left(\tau S_{\infty}, \tau S_{-\infty}^{\prime}\right) \stackrel{\sim}{\longrightarrow} \operatorname{Ext}\left(S_{\infty}, S_{-\infty}^{\prime}\right)
$$

since $\operatorname{Hom}\left(S_{\infty}, G\right)=0$. Thus $\operatorname{Ext}\left(S_{\infty}, S_{-\infty}^{\prime}\right) \neq 0$ follows since there is a nonzero map $\tau S_{\infty} \rightarrow S_{\infty}^{\prime}$.

Now suppose $N=G$ or $N=S_{\infty}$ for some quasi-simple $S$. Then $\operatorname{Ext}(M, N)$ $=0$ by Lemma 2.6 since $S_{\infty}^{\prime} \in \underline{\lim } \mathcal{R}$ and $S_{-\infty}^{\prime} \in \underline{\lim } \mathcal{P}$ for each quasi-simple $S^{\prime}$. For $M=G$, use the exact sequence $(2.1)$.

Finally, let $N=S_{-\infty}$. We have $\operatorname{Ext}\left(S_{-\infty}^{\prime}, N\right)=0$ for each quasi-simple $S^{\prime}$ since $\mathcal{P} \subseteq{ }^{\perp} N$. Now assume $S^{\prime} \nsim S$. Then $\operatorname{Ext}\left(\tau^{i} S^{\prime}, S_{-\infty}\right)=0$ for all $i \geq 0$ by Lemma 4 in [10]. Therefore $\operatorname{Ext}\left(S_{n}^{\prime}, S_{-\infty}\right)=0$ for all $n \geq 1$. Thus $\operatorname{Ext}\left(S_{\infty}^{\prime}, N\right) \cong \lim \operatorname{Ext}\left(S_{n}^{\prime}, N\right)=0$. Using again the exact sequence (2.1),

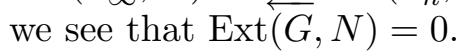

We need also a statement which involves finitely generated modules.

Lemma 2.8. Let $S$ and $S^{\prime}$ be quasi-simples such that $S \neq S^{\prime}$. Then $\operatorname{Ext}\left(S_{n}, S_{m}^{\prime}\right)=0$ for all $n, m \in \mathbb{N} \cup\{-\infty, \infty\}$.

Proof. Observe that for each $n \in \mathbb{N}$ the module $S_{n}$ is a submodule of $S_{\infty}$ and a quotient of $S_{-\infty}$. Now the assertion follows from Lemma 2.7 since $\operatorname{Ext}(-,-)$ is right exact.

\section{Cotilting modules.}

3.1. A characterization. In this section we prove our main result about cotilting modules for tame hereditary algebras. First we show that cotilting modules are precisely the maximal objects among all selforthogonal modules. This observation leads to some useful characterizations. 
Proposition 3.1. The following are equivalent for a pure-injective $\Lambda$-module $T$ :

(1) $T$ is a cotilting module;

(2) $T$ is maximal selforthogonal.

Proof. (1) $\Rightarrow(2)$ : First observe that that ${ }^{\perp} T \subseteq$ Cogen $T$. To see this, denote by $Q$ an injective cogenerator for $\operatorname{Mod} \Lambda$ and let $X$ be a module in ${ }^{\perp} T$. There is a monomorphism $X \rightarrow Q^{\alpha}$. Consider the pullback

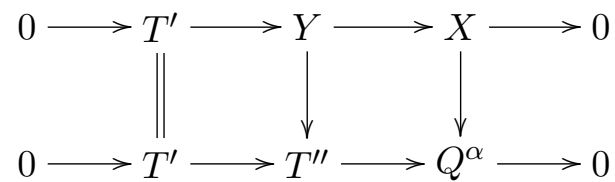

with $T^{\prime}, T^{\prime \prime}$ in $\operatorname{Prod} T$. The upper sequence splits and we obtain a monomorphism $X \rightarrow T^{\prime \prime}$.

Now assume $X$ is a module such that $T \amalg X$ is selforthogonal. Since $X$ is in $\operatorname{Cogen} T$, we can choose a left $\operatorname{Prod} T$-approximation of $X$, to get an exact sequence

$$
\xi: 0 \longrightarrow X \longrightarrow T^{\prime} \longrightarrow Y \longrightarrow 0
$$

that remains exact when one applies $\operatorname{Hom}(-, T)$ to it. Therefore one gets that $Y$ is also in ${ }^{\perp} T$. Then there is an exact sequence

$$
0 \longrightarrow Y \longrightarrow T^{\prime \prime} \longrightarrow Z \longrightarrow 0
$$

with $T^{\prime \prime} \in \operatorname{Prod} T$. We apply $\operatorname{Hom}(-, X)$ to this sequence and obtain $\operatorname{Ext}(Y, X)=0$ since id $X \leq 1$. Thus $\xi$ splits. This means $X$ belongs to $\operatorname{Prod} T$, so $T$ is maximal selforthogonal.

$(2) \Rightarrow(1)$ : First observe that ${ }^{\perp} T$ is closed under taking products by Corollary 1.10. Now apply Lemma 1.11.

Having shown that cotilting modules are maximal selforthogonal modules, we can apply our results about selforthogonal modules. First we separate the finitely generated cotilting modules from those having indecomposable summands which are not finitely generated.

Proposition 3.2. Let $T$ be a cotilting module with a finitely generated preprojective or preinjective direct summand. Then $T$ is equivalent to a finitely generated cotilting module.

Proof. Apply Lemma 2.5.

Next we introduce for each tube $\mathcal{T}$ in the category of regular modules the $\mathcal{T}$-component of a module and use this to characterize the cotilting modules.

Let $\mathbb{X}=\mathbb{P} / \sim$ be the set of equivalence classes of quasi-simples. Note that we obtain a decomposition $\mathcal{R}=\coprod_{\sigma \in \mathbb{X}} \mathcal{T}_{\sigma}$ of $\mathcal{R}$ into connected abelian categories, where each quasi-simple $S$ belongs to $\mathcal{T}_{\sigma}$ for $\sigma=[S]$. The categories $\mathcal{T}_{\sigma}$ are usually called tubes and card $\sigma$ is called the rank of the tube 
$\mathcal{T}_{\sigma}$. We say that a pure-injective module $M$ belongs to a tube $\mathcal{T}$ if every indecomposable direct summand of $M$ is of the form $S_{n}$ with $S \in \mathcal{T}$ and $n \in \mathbb{N} \cup\{-\infty, \infty\}$. The subcategory formed by all modules belonging to $\mathcal{T}$ is denoted by $\overline{\mathcal{T}}$. Given a pure-injective module $M$ and a tube $\mathcal{T}$, we define the $\mathcal{T}$-component $M_{\mathcal{T}}$ of $M$ to be a maximal direct summand of $M$ belonging to $\overline{\mathcal{T}}$. Note that $M_{\mathcal{T}}$ is unique up to isomorphism.

Proposition 3.3. Let $T$ be a pure-injective module without a nonzero finitely generated preprojective or preinjective direct summand. Then the following are equivalent:

(1) $T$ is a cotilting module;

(2) each $T_{\mathcal{T}}$ is maximal selforthogonal among all modules in $\overline{\mathcal{T}}$.

Proof. (1) $\Rightarrow(2)$ : We know from Proposition 3.1 that $T$ is maximal selforthogonal. In particular, each $T_{\mathcal{T}}$ is selforthogonal. Assume there is a tube $\mathcal{T}$ such that $T_{\mathcal{T}}$ is not maximal selforthogonal. Then there exists $X$ in $\overline{\mathcal{T}}$ with $X$ not in $\operatorname{Prod} T_{\mathcal{T}}$ such that $T_{\mathcal{T}} \amalg X$ is selforthogonal. The module $X$ has no extensions with the direct summands of $T$ belonging to different tubes, by Lemma 2.8. It follows from Corollary 2.3 that $T \amalg X$ is selforthogonal in $\operatorname{Mod} \Lambda$, since $T$ has no preprojective or preinjective summands. This is a contradiction.

$(2) \Rightarrow(1)$ : First observe that $T$ has an indecomposable direct summand which is not finitely generated. This follows from the fact that for each tube $\mathcal{T}$ of rank $1, T_{\mathcal{T}}$ has no finitely generated summands.

Next we need to show that $T$ is selforthogonal. By Corollary 2.3, it is sufficient to show $\operatorname{Ext}\left(T^{\prime}, T^{\prime \prime}\right)=0$ for indecomposable direct summands $T^{\prime}, T^{\prime \prime}$ of $T$. If $T^{\prime}$ and $T^{\prime \prime}$ belong to different tubes, then it follows from Lemma 2.8 that $\operatorname{Ext}\left(T^{\prime}, T^{\prime \prime}\right)=0$. If they belong to the same tube, then $\operatorname{Ext}\left(T^{\prime}, T^{\prime \prime}\right)=0$ by the assumption on $T_{\mathcal{T}}$.

Assume $T$ is not a cotilting module. Using Lemma 1.11, we find a pureinjective module $X$ such that $T \amalg X$ is a cotilting module. Choose an indecomposable direct summand $X^{\prime}$ of $X$ which does not belong to $\operatorname{Prod} T$. It follows from Proposition 3.2 that $X^{\prime}$ is neither preprojective nor preinjective since $T$ has an indecomposable summand which is not finitely generated. Thus $X^{\prime}$ belongs to a tube $\mathcal{T}$. It follows that $T_{\mathcal{T}} \amalg X^{\prime}$ is a selforthogonal module in $\overline{\mathcal{T}}$. A contradiction.

Remark 3.4. Given a tube $\mathcal{T}$, one can show that the modules in $\overline{\mathcal{T}}$ are precisely the filtered limits and the filtered colimits of modules in $\mathcal{T}$.

3.2. Selforthogonal modules in tubes. Fix a tube $\mathcal{T}$ of rank $r$. We say that a pure-injective module $M$ belonging to $\mathcal{T}$ is of Prüfer type if $M$ has no adic module as a direct summand. Analogously, $M$ is of adic type if $M$ has no Prüfer module as a direct summand. Next we define a bijection $M \mapsto M^{\vee}$ 
between the modules of Prüfer and adic type in $\overline{\mathcal{T}}$. To this end number the quasi-simples $S(1), \ldots, S(r)$ in $\mathcal{T}$ such that $\tau^{j} S(i)=S(i+j)$ for $i, j \in \mathbb{Z}_{r}$. If $M=S(i)_{n}$ is indecomposable, let $M^{\vee}=S(-i)_{-n}$. If $M=E\left(\coprod_{i} M_{i}\right)$ with all $M_{i}$ indecomposable, let $M^{\vee}=E\left(\coprod_{i} M_{i}^{\vee}\right)$.

Lemma 3.5. The map $M \mapsto M^{\vee}$ induces a bijection between the modules of Prüfer type and the modules of adic type in $\overline{\mathcal{T}}$. Moreover, $M$ is selforthogonal if and only if $M^{\vee}$ is selforthogonal.

Proof. It follows immediately from the definition that $M^{\vee \vee} \cong M$ for all $M \in \overline{\mathcal{T}}$. Therefore $M \mapsto M^{\vee}$ induces a bijection between the modules of Prüfer and adic type.

The rest follows from Lemma 3.6 given below. In addition, we use Corollary 2.3 which says that a pure-injective module $M$ is selfortogonal if $\operatorname{Ext}\left(M^{\prime}, M^{\prime \prime}\right)=0$ for all indecomposable direct summands $M^{\prime}, M^{\prime \prime}$ of $M$.

Lemma 3.6. Let $i, j \in \mathbb{Z}_{r}$ and $n, m \in \mathbb{N} \cup\{\infty\}$. Then $\operatorname{Ext}\left(S(i)_{n}, S(j)_{m}\right)=$ 0 if and only if $\operatorname{Ext}\left(S(-j)_{-m}, S(-i)_{-n}\right)=0$.

Proof. First observe that $S_{-n}=D\left((D S)_{n}\right)$ for any quasi-simple $S$. The assertion follows from the formula $\operatorname{Ext}(D M, D N) \cong \operatorname{Ext}\left(N, D^{2} M\right)$ which is valid for all modules $M$ and $N$; it is a consequence of the formula $\operatorname{Ext}(M, D N) \cong \operatorname{Ext}(N, D M)$. In addition, one uses that $D^{2}\left(S_{\infty}\right)$ is a coproduct of copies of $S_{\infty}$.

Each tube $\mathcal{T}$ gives rise to a locally finite Grothendieck category $\lim \mathcal{T}$. Using Theorem 1.13, we can classify its cotilting objects and get a description of the selforthogonal modules of Prüfer type. This leads to the following result which is taken from $[\mathbf{6}]$ :

Proposition 3.7. Let $M$ be a selforthogonal module of Prüfer type belonging to a tube $\mathcal{T}$. Then $M$ is maximal among all selforthogonal modules in $\overline{\mathcal{T}}$ if and only if the number of indecomposable non-isomorphic direct summands of $M$ equals the rank of $\mathcal{T}$.

Proposition 3.7 extends to arbitrary selforthogonal modules belonging to tubes.

Corollary 3.8. Let $M$ be a selforthogonal module belonging to a tube $\mathcal{T}$. Then $M$ is maximal among all selforthogonal modules in $\overline{\mathcal{T}}$ if and only if the number of indecomposable non-isomorphic direct summands of $M$ equals the rank of $\mathcal{T}$.

Proof. Each selforthogonal module is of Prüfer or of adic type. This follows from Lemma 2.7. Now use Proposition 3.7 and Lemma 3.5. 
3.3. The main theorem. We are now in the position to prove our main result about tame hereditary algebras.

Theorem 3.9. Let $\Lambda$ be a tame hereditary algebra and let $T$ be a pureinjective $\Lambda$-module.

(1) Suppose all indecomposable direct summands are finitely generated. Then $T$ is a cotilting module if and only if the number of non-isomorphic indecomposable direct summands of $T$ equals the number of simple $\Lambda$-modules and $\operatorname{Ext}\left(T^{\prime}, T^{\prime \prime}\right)=0$ for all $T^{\prime}, T^{\prime \prime} \in \operatorname{indec} T$.

(2) Suppose there is an indecomposable direct summand which is not finitely generated. Then $T$ is a cotilting module if and only if the following hold:

- Each indecomposable direct summand of $T$ is either generic or of the form $S_{n}$ for some $S \in \mathbb{P}$ and some $n \in \mathbb{N} \cup\{-\infty, \infty\}$.

- For each tube $\mathcal{T}$, let $I_{\mathcal{T}}$ be the set of non-isomorphic indecomposable direct summands of $T$ which are of the form $S_{n}$ for some $n \in \mathbb{N} \cup$ $\{-\infty, \infty\}$ and some quasi-simple $S \in \mathcal{T}$. Then card $I_{\mathcal{T}}$ equals the rank of $\mathcal{T}$ and $\operatorname{Ext}\left(T^{\prime}, T^{\prime \prime}\right)=0$ for all $T^{\prime}, T^{\prime \prime} \in I_{\mathcal{T}}$.

(3) Two cotilting modules $T_{1}$ and $T_{2}$ are equivalent if and only if $\operatorname{indec}\left(T_{1} \amalg\right.$ $G)=\operatorname{indec}\left(T_{2} \amalg G\right)$.

Proof. (1) This is a well-known result from [4].

(2) Assume first $T$ is a cotilting module with a summand which is not finitely generated. Then there are no preprojective or preinjective direct summands, by Proposition 3.2. It follows that all direct summands of $T$ are either generic or of the form $S_{n}$ for some quasi-simple $S \in \mathbb{P}$ and some $n \in \mathbb{N} \cup\{-\infty, \infty\}$. Now fix a tube $\mathcal{T}$ and consider the $\mathcal{T}$-component $T_{\mathcal{T}}$ of $T$. Proposition 3.3 implies that $T_{\mathcal{T}}$ is maximal selforthogonal among all modules in $\overline{\mathcal{T}}$. Thus card $I_{\mathcal{T}}$ equals the rank of $\mathcal{T}$ by Corollary 3.8.

Suppose now $T$ is a module such that each direct summand of $T$ is either generic or of the form $S_{n}$ for some quasi-simple $S \in \mathbb{P}$ and some $n \in \mathbb{N} \cup\{-\infty, \infty\}$. Suppose also that card $I_{\mathcal{T}}$ equals the rank of $\mathcal{T}$ and $\operatorname{Ext}\left(T^{\prime}, T^{\prime \prime}\right)=0$ for all $T^{\prime}, T^{\prime \prime} \in I_{\mathcal{T}}$. It follows from Corollary 2.3 that $T_{\mathcal{T}}$ is selforthogonal for each tube $\mathcal{T}$. Moreover, Corollary 3.8 implies that $T_{\mathcal{T}}$ is maximal selforthogonal among all modules in $\overline{\mathcal{T}}$. Thus $T$ is a cotilting module by Proposition 3.3.

(3) Recall the well-known fact that any finitely generated indecomposable module $X$ arises as a direct summand of a product $\prod_{i} M_{i}$ if $X$ is a direct summand of one of the $M_{i}$. It is therefore sufficient to consider statement (3) in case $T_{1}$ and $T_{2}$ are both not finitely generated. All one needs to show is that the generic module $G$ is the only indecomposable module not in indec $T$ that can occur in Prod $T$, for a cotilting module $T$ which is not finitely generated. Let $T_{1} \sim T_{2}$ and suppose $M$ is in indec $T_{2}$, but not in indec $T_{1}$. Then $T=M \amalg T_{1}$ is cotilting. Assume $M$ is not generic. Then 
there is a tube $\mathcal{T}$ such that $M$ is in $\overline{\mathcal{T}}$. It follows that $T_{\mathcal{T}}$ is not selforthogonal. This contradiction shows $M$ is generic.

For the Kronecker algebra, there are no finitely generated selforthogonal regular modules. We obtain therefore the following description of all cotilting modules, up to equivalence:

Corollary 3.10. Let $T$ be a cotilting module over a Kronecker algebra. Then $T$ is equivalent to one of the following:

- A finitely generated preinjective cotilting module,

- a finitely generated preprojective cotilting module,

- a cotilting module of the form

$$
\left(\coprod_{S \in \mathbb{P}^{\prime}} S_{\infty}\right) \amalg E\left(\coprod_{S \in \mathbb{P}^{\prime \prime}} S_{-\infty}\right)
$$

for some partition $\mathbb{P}^{\prime} \cup \mathbb{P}^{\prime \prime}$ of $\mathbb{P}$.

\section{The maximal selforthogonal objects in tubes.}

Let us complete the characterization of the cotilting modules over a tame hereditary algebra. To this end we describe the maximal selforthogonal modules belonging to a tube. In [6], a complete combinatorial description of the maximal selforthogonal objects of Prüfer type is given. Using Lemma 3.5, one also gets a description of the maximal selforthogonal objects of adic type.

Let $\mathcal{T}$ be a tube of rank $r$ in $\operatorname{Mod} \Lambda$. Let $S(1), S(2), \ldots, S(r)$ be the quasisimples, ordered such that $\tau^{j} S(i)=S(i+j)$ for $i, j \in \mathbb{Z}_{r}$. The ray vector of a module $M$ in $\overline{\mathcal{T}}$ is an $r$-tuple $\left(a_{1}, a_{2}, \ldots, a_{r}\right)$ of nonnegative integers such that $M$ has exactly $a_{i}$ indecomposable direct summands with quasi-socle $S(i)$.

Proposition 4.1. Let $\mathcal{T}$ be a tube of rank $r$. For each $r$-tuple $\left(a_{1}, a_{2}, \ldots, a_{r}\right)$ of nonnegative integers with $\sum_{i} a_{i}=r$, there is exactly one maximal selforthogonal object $T$ of Prüfer type in $\overline{\mathcal{T}}$ with this $m$-tuple as its ray vector.

We refer to [6] for the proof; it also gives an algorithm how to find a maximal selforthogonal object with a given ray-vector.

Given a module $M$ in $\overline{\mathcal{T}}$, the coray vector of $M$ is an $r$-tuple $\left(b_{1}, b_{2}, \ldots, b_{r}\right)$, where $b_{i}$ is the number of indecomposable direct summands in $M$ with quasitop isomorphic to $S(i)$.

Proposition 4.2. Let $\mathcal{T}$ be a tube of rank $r$. For each $r$-tuple $\left(b_{1}, b_{2}, \ldots, b_{r}\right)$ of nonnegative integers with $\sum_{i} b_{i}=m$, there is exactly one maximal selforthogonal object $T$ of adic type in $\overline{\mathcal{T}}$, with this $r$-tuple as its coray vector. 
Proof. The correspondence $M \mapsto M^{\vee}$ assigns to a maximal selforthogonal object of Prüfer type and with ray vector $\left(a_{1}, a_{2}, \ldots, a_{r}\right)$, a maximal selforthogonal object of adic type and with coray vector $\left(a_{r-1}, a_{r-2}, \ldots, a_{r}\right)$.

Acknowledgments. We would like to thank Helmut Lenzing, Claus M. Ringel and Øyvind Solberg for helpful comments and ideas. In addition, we are grateful to Lidia Angeleri Hügel for pointing out a mistake in one of our arguments.

\section{References}

[1] H.L. Angeleri and F.-U. Coelho, Infinitely generated complements to partial tilting modules, Math. Proc. Cambridge Phil. Soc., 132 (2002), 89-96, CMP 1866326.

[2] H.L. Angeleri, A. Tonolo and J. Trlifaj, Tilting preenvelopes and cotilting precovers, Algebr. Represent. Theory, 4 (2001), 155-170, MR 2002e:16010, Zbl 0999.16007.

[3] M. Auslander and I. Reiten, Applications of contravariantly finite subcategories, Adv. Math., 86 (1991), 111-152, MR 92e:16009, Zbl 0774.16006.

[4] K. Bongartz, Tilted algebras, in 'Representations of algebras (Puebla, 1980),' Lecture Notes in Math., 903, Springer, Berlin-New York, 1981, 26-38, MR 83g:16053, Zbl 0478.16025.

[5] S. Brenner and M.C.R. Butler, Generalizations of the Bernstein-Gelfand-Ponomarev reflection functors, in 'Representation theory, II' (Proc. Second Internat. Conf., Carleton Univ., Ottawa, Ont., 1979), Lecture Notes in Math., 832, Springer, Berlin-New York, 1980, 103-169, MR 83e:16031, Zbl 0446.16031.

[6] A.B. Buan and H. Krause, Tilting and cotilting for quivers of type $\widetilde{A}_{n}$. Preprint, 2002.

[7] R. Colpi, G. D'Este and A. Tonolo, Quasi-tilting modules and counter equivalences, J. Algebra, 191 (1997), 461-494, MR 99g:16008, Zbl 0876.16004.

[8] R. Colpi, A. Tonolo and J. Trlifaj, Partial cotilting modules and the lattice induced by them, Comm. Algebra, 25 (1997), 3225-3237, MR 98i:16003, Zbl 0893.16017.

[9] W.W. Crawley-Boevey, Locally finitely presented additive categories, Comm. Algebra, 22 (1994), 1644-1674, MR 95h:18009, Zbl 0798.18006.

[10] _ Infinite-dimensional modules in the representation theory of finite-dimensional algebras, CMS Conf. Proc., 23 (1998), 29-54, MR 99m:16016, Zbl 0920.16007.

[11] E. Enochs, Injective and flat covers, envelopes and resolvents, Israel J. Math., 39 (1981), 189-209, MR 83a:16031, Zbl 0464.16019.

[12] G. Gabriel, Des catégories abéliennes, Bull. Soc. Math. France, 90 (1962), 323-448, MR 38 \#1144, Zbl 0201.35602.

[13] W. Geigle, The Krull-Gabriel dimension of the representation theory of a tame hereditary Artin algebra and applications to the structure of exact sequences, Manuscripta Math., 54 (1985), 83-106, MR 87e:16061, Zbl 0593.16022.

[14] R. Göbel and J. Trlifaj, Cotilting and a hierarchy of cotorsion groups, J. Algebra, 224 (2000), 110-122, MR 2001a:20100, Zbl 0947.20036.

[15] C. Jensen and H. Lenzing, Model-Theoretic Algebra with Particular Emphasis on Fields, Rings, Modules, Algebra, Logic and Applications, 2, Gordon and Breach Science Publishers, New York, 1989, MR 91m:03038, Zbl 0728.03026. 
[16] H. Krause and $\varnothing$. Solberg, Filtering modules of finite projective dimension, Forum Math., to appear.

[17] H. Lenzing, Homological transfer from finitely presented to infinite modules, in 'Abelian group theory (Honolulu, Hawaii, 1983),' Lecture Notes in Math., 1006, Springer, Berlin, 1983, 734-761, MR 85f:16034, Zbl 0529.16016.

[18] C.M. Ringel, Infinite-dimensional representations of finite-dimensional hereditary algebras, Symposia Mathematica, Vol. XXIII (Conf. Abelian Groups and their Relationship to the Theory of Modules, INDAM, Rome, 1977), Academic Press, London-New York, 1979, 321-412, MR 81i:16032 , Zbl 0429.16022.

[19] J. Rotman, An Introduction to Homological Algebra, Pure and Applied Mathematics, 85, Academic Press, Inc., New York-London, 1979, MR 80k:18001, Zbl 0441.18018.

Received April 8, 2002 and revised November 6, 2002.

InStitutT FOR MATEMATISKE FAG

NTNU

N-7491 TRONDHEIM

NORWAY

E-mail address: aslakb@math.ntnu.no

Department of Pure Mathematics

UNIVERSITY OF LEEDS

LEEDS LS2 9JT

UNiTED KINGDOM

E-mail address: henning@maths.leeds.ac.uk 